\title{
Factors related to outcomes in lupus-related protein-losing enteropathy
}

\author{
Doo-Ho Lim, Yong-Gil Kim, Seung-Hyeon Bae, Soomin Ahn, Seokchan Hong, Chang-Keun Lee, and Bin Yoo
}

Division of Rheumatology, Department of Internal Medicine, Asan Medical Center, University of Ulsan College of Medicine, Seoul, Korea

Received: November 1, 2014 Revised : November 21, 2014 Accepted: December 1, 2014

\section{Correspondence to}

Yong-Gil Kim, M.D.

Division of Rheumatology,

Department of Internal

Medicine, Asan Medical Center, University of Ulsan College of

Medicine, 88 Olympic-ro 43-gil,

Songpa-gu, Seoul 05505, Korea

Tel: +82-2-3010-3279

Fax: +82-2-3010-6969

E-mail: bestmd2000@amc.seoul.kr
Background/Aims: Protein-losing enteropathy (PLE), characterized by severe hypoalbuminemia and peripheral edema, is a rare manifestation of systemic lupus erythematosus. This present study aimed to identify the distinctive features of lupus-related PLE and evaluate the factors related to the treatment response.

Methods: From March 1998 to March 2014, the clinical data of 14 patients with lupus PLE and seven patients with idiopathic PLE from a tertiary center were reviewed. PLE was defined as a demonstration of protein leakage from the gastrointestinal tract by either technetium 99m-labelled human albumin scanning or fecal $\alpha 1$-antitrypsin clearance. A positive steroid response was defined as a return of serum albumin to $\geq 3.0 \mathrm{~g} / \mathrm{dL}$ within 4 weeks after initial steroid monotherapy, and remission as maintenance of serum albumin $\geq 3.0 \mathrm{~g} / \mathrm{dL}$ for at least 3 months. A high serum total cholesterol level was defined as a level of $\geq 240 \mathrm{mg} / \mathrm{dL}$.

Results: The mean age of the lupus-related PLE patients was 37.0 years, and the mean follow-up duration was 55.8 months. Significantly higher erythrocyte sedimentation rate and serum total cholesterol levels were found for lupus PLE than for idiopathic PLE. Among the 14 patients with lupus PLE, eight experienced a positive steroid response, and the serum total cholesterol level was significantly higher in the positive steroid response group. A positive steroid response was associated with an initial high serum total cholesterol level and achievement of remission within 6 months.

Conclusions: In lupus-related PLE, a high serum total cholesterol level could be a predictive factor for the initial steroid response, indicating a good response to steroid therapy alone.

Keywords: Protein-losing enteropathies; Lupus erythematosus, systemic; Hypercholesterolemia

\section{INTRODUCTION}

Protein-losing enteropathy (PLE) is characterized by an excessive loss of serum proteins into the gastrointestinal tract leading to hypoalbuminemia and peripheral edema. PLE can be caused by various diseases, such as inflammatory bowel disease, sarcoidosis, intestinal malignancy, infectious diseases of the gastrointestinal tract, cardiac diseases, and autoimmune diseases such as systemic lupus erythematosus (SLE) [1].

Lupus-related PLE (lupus PLE) is a rare manifestation of SLE. Hence, most previous reports involved isolated cases or small series of patients, making it difficult to establish the clinical or laboratory features, proper management, and prognosis. In this study, based on the experience accumulated since our previous report [2], 
we have attempted to identify the distinctive features of lupus PLE compared with idiopathic PLE, and verify the factors involved in the treatment response or outcome of lupus PLE.

\section{METHODS}

We reviewed the electronic records of 34 patients who received a diagnosis of PLE in a tertiary hospital in South Korea from March 1998 to March 2014. Among these 34 patients, 14 cases were diagnosed with SLE according to the 1997 revised American College of Rheumatology criteria for the classification of SLE [3] (the lupus PLE group), whereas an underlying cause was not identified in seven patients (i.e., the idiopathic PLE group) (Fig. 1). The other 13 cases of PLE were associated with Crohn's disease $(n=1)$, infectious enteritis $(n=4)$, amyloidosis $(n=2)$, colon cancer $(n=1)$, liver cirrhosis ( $n$ $=3$ ), and heart failure $(n=2)$. The diagnosis of PLE was made using a combination of the following: (1) protein leakage from the gastrointestinal tract by either technetium 99m-labelled human albumin scanning or fecal ג1-antitrypsin clearance; (2) no evidence of a significant loss of protein from other sources; and (3) no evidence of reduced protein synthesis such as severe liver disease or large liver resection.

Clinical information was collected within the follow-

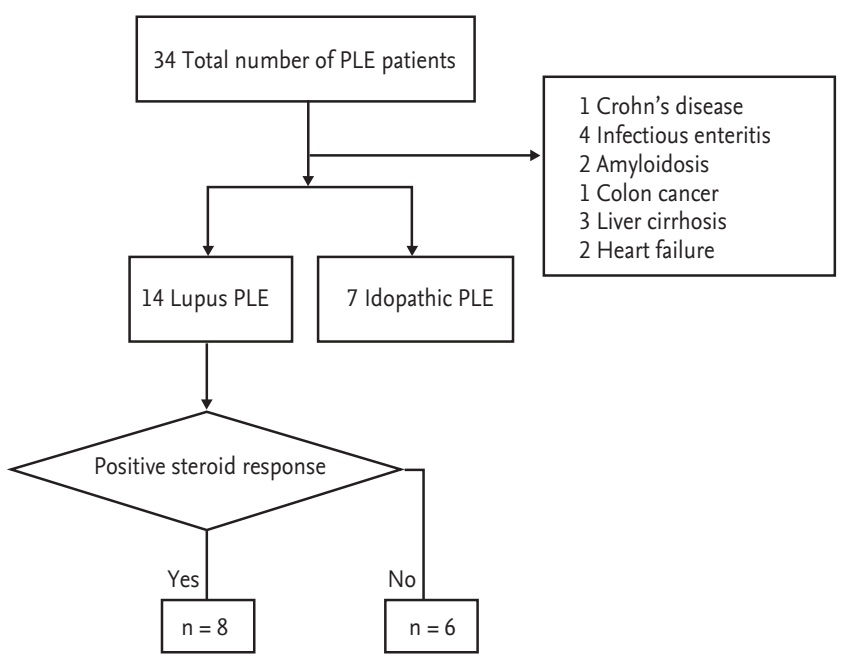

Figure 1. Overview of the protein losing enteropathy (PLE) patients in this study. ing areas: demographic characteristics, clinical features, laboratory data, radiological imaging, and histological features from a bowel biopsy. For the lupus PLE patients, autoantibody profiles (anti-double stranded DNA antibodies, anti-nuclear antibodies, and anti-extractable nuclear antibodies), lupus anticoagulant complements, and the SLE disease activity index were additionally obtained at the time of PLE diagnosis. A positive steroid response was determined if the serum albumin level returned to $\geq 3.0 \mathrm{~g} / \mathrm{dL}$ within 4 weeks of initial steroid therapy. Remission was defined as a maintained serum albumin level of $\geq 3.0 \mathrm{~g} / \mathrm{dL}$ for at least 3 months. High serum total cholesterol was a serum total cholesterol level of $\geq 240 \mathrm{mg} / \mathrm{dL}$.

Lupus PLE patients were initially treated with intravenous (IV) methylprednisolone $1 \mathrm{mg} / \mathrm{kg} /$ day and switched to oral prednisolone. If they obtained a positive steroid response, prednisolone was tapered off slowly over a period of 10 months. If a positive steroid response was not achieved, another immunosuppressive therapy such as monthly IV cyclophosphamide pulse therapy (500 to $750 \mathrm{mg} / \mathrm{m}^{2} / \mathrm{month}$ ) or oral azathioprine 50 to $100 \mathrm{mg} /$ day was combined with the prednisolone treatment.

Data were analyzed with SPSS version 18.0 (SPSS, Inc., Chicago, IL, USA). All continuous variables were expressed as the mean $\pm \mathrm{SD}$. Categorical variables were reported as numbers and percentages. Continuous variables were compared using the Mann-Whitney $U$ test, and categorical variables were compared using the chisquare test or a Fisher exact test. Statistical significance was defined as a two tailed $p$ value of less than 0.05 .

This study fulfilled the ethical guidelines of the Declaration of Helsinki (Edinburgh 2000) and received approval from the Institutional Review Board of the Asan Medical Center.

\section{RESULTS}

The baseline characteristics of lupus PLE and idiopathic PLE in our study patients are indicated in Table 1. Patients with lupus PLE tended to be younger and were more likely to be female than patients with idiopathic PLE. In our lupus PLE patients, the white blood cell count, erythrocyte sedimentation rate (ESR), and serum total cholesterol levels were significantly increased $(p<$ 
Table 1. Baseline characteristics of the lupus PLE and idiopathic PLE patients in this study

\begin{tabular}{|c|c|c|c|}
\hline Characteristic & Lupus PLE $(\mathrm{n}=14)$ & Idiopathic PLE $(\mathrm{n}=7)$ & $p$ value \\
\hline Age, yr & $41.9 \pm 16.3$ & $51.3 \pm 16.8$ & 0.231 \\
\hline Male sex & $6(42.6)$ & $5(71.4)$ & 0.361 \\
\hline Alcohol & $4(28.6)$ & $4(57 \cdot 1)$ & 0.346 \\
\hline Smoking & $4(28.6)$ & $3(42.9)$ & 0.638 \\
\hline Peripheral edema & $14(100)$ & $7(100)$ & 1.000 \\
\hline Abdominal pain & $1(7.1)$ & $1(14 \cdot 3)$ & 1.000 \\
\hline Diarrhea & $6(42.6)$ & $5(71.4)$ & 0.361 \\
\hline Ascites & $12(85 \cdot 7)$ & $3(42.9)$ & 0.120 \\
\hline Pleural effusion & $10(71.4)$ & $5(71.4)$ & 1.000 \\
\hline Pericardial effusion & $4(28.6)$ & o & 0.018 \\
\hline White blood cells, $\times 10^{3} / \mu \mathrm{L}$ & $7 \cdot 4 \pm 2.7$ & $4.1 \pm 1.4$ & 0.002 \\
\hline Lymphocyte, $\times 10^{3} / \mu \mathrm{L}$ & $1.7 \pm 0.7$ & $1.1 \pm 0.8$ & 0.094 \\
\hline Hemoglobin, g/dL & $12.2 \pm 1.4$ & $11.2 \pm 1.7$ & 0.097 \\
\hline Platelets, $\times 10^{3} / \mu \mathrm{L}$ & $227 \cdot 3 \pm 114.2$ & $273.6 \pm 71.6$ & 0.360 \\
\hline Erythrocyte sedimentation rate, $\mathrm{mm} / \mathrm{hr}$ & $69.4 \pm 37.2$ & $9.1 \pm 10.6$ & 0.001 \\
\hline C-reactive protein, $\mathrm{mg} / \mathrm{dL}$ & $1.1 \pm 1.6$ & $1.2 \pm 1.6$ & 1.000 \\
\hline Protein, g/dL & $4.2 \pm 1.3$ & $4.0 \pm 0.8$ & 0.899 \\
\hline Albumin, g/dL & $1.2 \pm 0.5$ & $1.6 \pm 0.5$ & 0.122 \\
\hline Total cholesterol level, mg/dL & $249.6 \pm 91.4$ & $82.7 \pm 34.7$ & $<0.001$ \\
\hline
\end{tabular}

Values are presented as mean \pm SD or number (\%).

PLE, protein losing enteropathy.

0.05). However, the lymphocyte, protein, albumin, and C-reactive protein (CRP) levels were not found to differ between the two groups.

Eight patients with lupus PLE and four patients with idiopathic PLE were diagnosed with PLE based on the technetium 99m-labelled human albumin scanning. Among these lupus PLE patients, the most common albumin leakage site was the small intestine $(n=4)$, followed by the small and large intestine $(n=2)$ and stom$\operatorname{ach}(n=2)$. Endoscopic biopsies were performed on the stomach, small intestine, or large bowel in 10 patients with lupus PLE and four patients with idiopathic PLE. For patients with human albumin scanning, biopsies were taken from the suspected albumin-leakage site (Table 2). Histological findings from the lupus PLE patients were mostly of non-specific abnormalities, including chronic inflammation with mild edema, except for one biopsy specimen from the jejunum, which showed marked blunting and atrophy of the jejunal villi (\#10, who was categorized as being in the negative steroid re- sponse group). Among the idiopathic PLE patients, two patients had villi edema with lymphatic dilatation while the other two patients showed non-specific chronic inflammation.

Among the lupus PLE patients, eight patients (57.1\%) were female and 11 patients (78.6\%) initially presented with PLE at the time when SLE was diagnosed. The mean follow-up duration for lupus PLE was $55.8 \pm 16.0$ months (range, 8 to 179). One patient had had a history of lupus nephritis class IV 2.5 years ago; however, her 24hour urine protein was less than $500 \mathrm{mg} /$ day at the time of PLE diagnosis.

As shown in Table 3, eight patients had a positive steroid response and serum total cholesterol levels were significantly elevated in the positive steroid response group compared to the negative steroid response group. However, there were no differences in other parameters, such as ESR, CRP, C3, C4, and albumin. A high serum total cholesterol level $(\geq 240 \mathrm{mg} / \mathrm{dL}$ ) was evident in seven out of the eight patients (87.5\%) in the positive steroid 
Table 2. Histological findings of specimens acquired from endoscopic biopsies

\begin{tabular}{|c|c|c|c|}
\hline & Biopsy lesion & Histological finding & Albumin scan (leakage site) \\
\hline \multicolumn{4}{|c|}{ Lupus PLE } \\
\hline \multirow[t]{2}{*}{$\# 1$} & Gastric antrum and body & Chronic gastritis & Distal small bowel \\
\hline & Terminal ileum & Minimal nonspecific inflammation & \\
\hline \multirow[t]{2}{*}{ \#2 } & Gastric antrum & Superficial gastritis with mild edema & $\begin{array}{l}\text { Distal small bowel and } \\
\text { ascending colon }\end{array}$ \\
\hline & Random colon biopsies & Mild edema & \\
\hline \multirow[t]{2}{*}{ \#3 } & Gastric antrum & Chronic gastritis & $\begin{array}{l}\text { Distal small bowel and } \\
\text { ascending colon }\end{array}$ \\
\hline & Random colon biopsies & Mild nonspecific colitis & \\
\hline \#4 & Gastric antrum & Mild chronic gastritis & Not done \\
\hline \#5 & Gastric antrum & Gastric mucosa with edema & Not done \\
\hline \#6 & Duodenum & Chronic duodenitis & Not done \\
\hline \multirow[t]{2}{*}{ \#7 } & Duodenum & Chronic inflammation & Proximal small bowel \\
\hline & Rectum & Chronic colitis & \\
\hline \#8 & Gastric antrum and body & Chronic gastritis & Stomach \\
\hline \#9 & Gastric antrum and body & Chronic active gastritis & Stomach \\
\hline$\# 10$ & Proximal jejunum & $\begin{array}{l}\text { Marked blunting and atrophy of villi } \\
\text { with edema }\end{array}$ & Not done \\
\hline \multicolumn{4}{|c|}{ Idiopathic PLE } \\
\hline \multirow[t]{2}{*}{$\# 1$} & Duodenum & Villi edema with lymphatic dilatation & Not done \\
\hline & Random colon biopsies & Moderate nonspecific colitis & \\
\hline \#2 & Duodenum & $\begin{array}{l}\text { Lymphatic dilatation with marked villous } \\
\text { atrophy and edema }\end{array}$ & Proximal small bowel \\
\hline \#3 & Gastric antrum & Chronic moderate inflammation & Not done \\
\hline \multirow[t]{2}{*}{ \#4 } & Duodenum & Chronic inflammation & Ascending colon \\
\hline & Random colon biopsies & Chronic inflammation with erosion & \\
\hline
\end{tabular}

Random colon biopsies: biopsies from terminal ileum, ascending, transverse, descending, sigmoid colon.

PLE, protein losing enteropathy.

response group, but not in any of the cases in the negative steroid response group. A high serum total cholesterol level was significantly associated with a positive steroid response (odds ratio [OR], 7.0; 95\% confidence interval [CI], 1.14 to 42.97 ; $p=0.005$ ). Moreover, a positive steroid response was related to remission within 6 months (OR, 3.0; $95 \%$ CI, 0.97 to 9.30; $p=0.015$ ). Irrespective of the treatment regimen, once lupus PLE patients underwent remission, the serum total cholesterol level, ESR, and CRP decreased and the complement level recovered (data not shown).

Among our lupus PLE cases, the 10 patients who achieved remission within 6 months showed a higher serum total cholesterol level $(283.3 \pm 79.3 \mathrm{mg} / \mathrm{dL})$ than the four patients who did not $(165.3 \pm 63.9 \mathrm{mg} / \mathrm{dL})$. Among these 10 patients, only one patient experienced a relapse 3 years after remission; this patient was treated again with prednisolone (1 mg/kg/day) only to obtain a positive steroid response and remission within 6 months (Table 4). Among the four patients who did not achieve remission within 6 months, three did not obtain remission during their follow-up duration of 10, 24, and 60 months, respectively. The other patient achieved remission at 13 months after a combination treatment with prednisolone and cyclophosphamide, but relapsed 6 years after remission. This patient subsequently achieved remis- 
Table 3. Clinical parameters related to the initial steroid response

\begin{tabular}{|c|c|c|c|}
\hline Characteristic & Positive steroid response $(\mathrm{n}=8)$ & Negative steroid response $(n=6)$ & $p$ value \\
\hline Age, yr & $37.0 \pm 19.8$ & $48.3 \pm 7.7$ & 0.106 \\
\hline Male sex & $4(50)$ & $2(33 \cdot 3)$ & 0.548 \\
\hline Symptom duration before treatment, wk & $6.1 \pm 4 \cdot 5$ & $16.0 \pm 11.5$ & 0.077 \\
\hline White blood cells, $\times 10^{3} / \mu \mathrm{L}$ & $7 \cdot 7 \pm 3 \cdot 3$ & $7.0 \pm 1.9$ & 0.897 \\
\hline Lymphocyte, $\times 10^{3} / \mu \mathrm{L}$ & $1.9 \pm 0.7$ & $1.5 \pm 0.5$ & 0.197 \\
\hline Hemoglobin, g/dL & $12.8 \pm 1.1$ & $11.5 \pm 1.5$ & 0.121 \\
\hline Platelets, $\times 10^{3} / \mathrm{L}$ & $202.8 \pm 119.9$ & $260.1 \pm 107.2$ & 0.302 \\
\hline Erythrocyte sedimentation rate, $\mathrm{mm} / \mathrm{hr}$ & $68.3 \pm 23.9$ & $70.7 \pm 51.2$ & 0.886 \\
\hline C-reactive protein, $\mathrm{mg} / \mathrm{dL}$ & $0.9 \pm 1.9$ & $1.1 \pm 1.0$ & 0.196 \\
\hline $\mathrm{C}_{3}, \mathrm{mg} / \mathrm{dL}$ & $52.8 \pm 24.6$ & $44 \cdot 3 \pm 19 \cdot 4$ & 0.606 \\
\hline $\mathrm{C}_{4}, \mathrm{mg} / \mathrm{dL}$ & $11.3 \pm 5.7$ & $13.2 \pm 3.2$ & 0.366 \\
\hline Anti-dsDNA antibody, IU/mL & $16.6 \pm 19.8$ & $20.4 \pm 41.5$ & 0.597 \\
\hline SLEDAI score & $6.5 \pm 3 \cdot 3$ & $7.67 \pm 3.7$ & 0.511 \\
\hline Protein, g/dL & $4.2 \pm 1.6$ & $4.23 \pm 0.9$ & 0.560 \\
\hline Albumin, g/dL & $1.3 \pm 0.5$ & $1.2 \pm 0.35$ & 0.755 \\
\hline Total cholesterol level, mg/dL & $304.6 \pm 74.0$ & $176.6 \pm 52.3$ & 0.008 \\
\hline High serum total cholesterol & $7(87.5)$ & o & 0.005 \\
\hline Remission within 6 months & $8(100)$ & $2(33 \cdot 3)$ & 0.015 \\
\hline
\end{tabular}

Values are presented as mean \pm SD or number (\%).

dsDNA, double stranded DNA; SLEDAI, systemic lupus erythematosus disease activity index.

sion within 6 months by initial combination treatment with both prednisolone and azathioprine.

\section{DISCUSSION}

In contrast to idiopathic PLE, an elevated serum total cholesterol level is relatively common in lupus PLE [4-9], possibly because the main mechanism underlying these conditions is different. In lymphangiectasia, which is considered to be the main pathogenesis of idiopathic PLE, most serum components, such as albumin, gamma globulin, cholesterol, and lymphocyte, can be decreased [10]. However, complement-mediated vascular injury or cytokines related to increased endothelial permeability have been regarded as a possible mechanism for lupus PLE, which allows a more selective leakage of small diameter particles such as albumin [2]. Moreover, hypoalbuminemia triggers hepatic lipoprotein synthesis and inhibits lipoprotein catabolism, resulting in severe hypoalbuminemia with hypercholesterolemia, as with similar mechanisms such as nephrotic syndrome [11]. In our current analyses, the serum total cholesterol level was found to be significantly higher in the lupus PLE patients, as also observed in a previous report [2]. In addition, regarding the pathologic features, lymphatic dilatation was not found in the patients with lupus PLE, whereas half of the patients in our study population with idiopathic PLE showed lymphatic dilatation.

In our present investigation we found that a high serum total cholesterol level was associated with an initial steroid response in patients with lupus PLE. This might suggest in the first instance that lupus PLE patients with a high serum total cholesterol level had less severe gastrointestinal barrier damage and, therefore, showed a better response to steroid treatment without other immunosuppressants. In addition, a short duration of symptoms appears to be associated with a favorable initial steroid response. As displayed in Table 3, the patients in the positive steroid response group were treated early (mean duration, 6.4 weeks) compared to those in the negative response group (16.0 weeks). A long duration 
Table 4. The management and outcome of the patients with lupus protein losing enteropathy

\begin{tabular}{lllll}
\hline & Steroid response & Immunosuppressant agent & Remission within 6 months & Relapse \\
\hline$\# 1$ & Positive steroid response & Not added & Yes & No \\
$\#$ \#2 & Positive steroid response & Not added & Yes & No \\
$\# 3$ & Positive steroid response & Not added & Yes & No \\
$\# 4$ & Positive steroid response & Not added & Yes & No \\
$\# 5$ & Positive steroid response & Not added & Yes & No \\
$\# 6$ & Positive steroid response & Not added & Yes & No \\
$\# 7$ & Positive steroid response & Not added & Yes & No \\
$\# 8$ & Positive steroid response & Not added & Yes & Yes \\
$\# 9$ & Negative steroid response & Cyclophosphamide & Yes & No \\
$\# 10$ & Negative steroid response & Cyclophosphamide & Yes & No \\
$\# 11$ & Negative steroid response & Cyclophosphamide & No (remission at 13 months) & Yes \\
$\# 12$ & Negative steroid response & Cyclophosphamide & No & No remission \\
$\# 13$ & Negative steroid response & Azathioprine & No & No remission \\
$\# 14$ & Negative steroid response & Azathioprine & No & No remission \\
\hline
\end{tabular}

of symptoms seems to indicate an inevitable exposure to inflammatory destruction over a long period of time, which can cause more severe or irreversible gastrointestinal barrier damage in lupus PLE.

In our lupus PLE patients, the lipid profiles were initially checked in only five cases (four from the positive steroid response group and one from the negative steroid response group). All lipid fractions, from high density lipoprotein (HDL) to triglyceride (TG), were reduced in the patients from the negative steroid response group (HDL, $24 \mathrm{mg} / \mathrm{dL}$ [normal range, $\geq 40$ ]; TG, $78 \mathrm{mg}$ / $\mathrm{dL}$ [normal range, $\leq 199])$. Among the four patients with a positive steroid response, three patients showed low HDL levels (13, 19, and $20 \mathrm{mg} / \mathrm{dL}$, respectively) but high TG levels $(215,249$, and $223 \mathrm{mg} / \mathrm{dL}$, respectively). This finding might be because TG is the main component of hypercholesterolemia in lupus PLE, which can be associated with a favorable treatment response.

Compared to the our previous report [2], the follow-up period in this study was extended and the number of lupus patients were increased so that the data could be properly analyzed through the use of statistical tools. Therefore, we consider that these changes make our previous results more convincing.

Our present study has some limitations. First, all of our subjects were recruited from a single tertiary center and the series was relatively small. Thus, a selection bias could have influenced our findings and our results will need to be confirmed in a future larger-sized, multicenter study. Second, many biopsy samples were obtained from the stomach, which is a limiting factor considering that most loss of protein occurred from the small intestine in the lupus PLE patients $[1,10]$. Two lupus PLE patients underwent gastric biopsies due to protein leakage from the stomach on the human albumin scan. The other gastric biopsies were mainly obtained from patients without a human albumin scan because it was not easy to perform random biopsies from the small intestine without prior evidence of protein leakage. Third, the biopsy specimens from our lupus PLE patients were not immunochemically stained, which makes it difficult to assess the immune-mediated process of this disease. Finally, we were not able to check the levels of cholesterol subtypes in all patients. As different cholesterol subtypes have different molecular weights, the levels of these subtypes may possibly explain the degree of lymphatic destruction or might show other findings that could aid our understanding of the pathogenesis of lupus PLE.

In conclusion, a high serum total cholesterol level could be a predictive factor of an initial steroid response, suggesting a good response to steroid therapy alone. Moreover, the early detection and treatment of PLE could also be associated with the initial steroid response. 


\section{KEY MESSAGE}

1. Elevated serum total cholesterol was characteristic of lupus protein-losing enteropathy.

2. Initial high serum total cholesterol could be a predictive factor for a favorable steroid response.

3. An initial favorable response to the steroids was associated with the achievement of remission within 6 months.

\section{Conflict of interest}

No potential conflict of interest relevant to this article was reported.

\section{Acknowledgments}

This research was supported by the Asan Institute for Life Science (2014-463).

\section{REFERENCES}

1. Al-Mogairen SM. Lupus protein-losing enteropathy (LUPLE): a systematic review. Rheumatol Int 2011;31:995-1001.

2. Kim YG, Lee CK, Byeon JS, et al. Serum cholesterol in idiopathic and lupus-related protein-losing enteropathy. Lupus 2008;17:575-579.

3. Hochberg MC. Updating the American College of Rheu- matology revised criteria for the classification of systemic lupus erythematosus. Arthritis Rheum 1997;40:1725.

4. Perednia DA, Curosh NA. Lupus-associated protein-losing enteropathy. Arch Intern Med 1990;150:1806-1810.

5. Tsutsumi A, Sugiyama T, Matsumura R, et al. Protein losing enteropathy associated with collagen diseases. Ann Rheum Dis 1991;50:178-181.

6. Wood ML, Foulds IS, French MA. Protein losing enteropathy due to systemic lupus erythematosus. Gut 1984;25:1013-1015.

7. Chung U, Oka M, Nakagawa Y, et al. A patient with protein-losing enteropathy associated with systemic lupus erythematosus. Intern Med 1992;31:521-524.

8. Zheng WJ, Tian XP, Li L, et al. Protein-losing enteropathy in systemic lupus erythematosus: analysis of the clinical features of fifteen patients. J Clin Rheumatol 2007;13:313316.

9. Turkcapar N, Ozyuncu N, Cinar K, et al. A case of systemic lupus erythematosus presenting with protein-losing enteropathy. Turk J Gastroenterol 2006;17:226-230.

10. Law ST, Ma KM, Li KK. The clinical characteristics of lupus related protein-losing enteropathy in Hong Kong Chinese population: 10 years of experience from a regional hospital. Lupus 2012;21:840-847.

11. Kaysen GA, Gambertoglio J, Felts J, Hutchison FN. Albumin synthesis, albuminuria and hyperlipemia in nephrotic patients. Kidney Int 1987;31:1368-1376. 